\title{
The Littlewood-Paley Theory: A Common Thread of Many Works in Nonlinear Analysis
}

Hajer Bahouri (Université Paris-Est Créteil, France)

In this article we present the Littlewood-Paley theory and illustrate the effectiveness of this microlocal analysis tool in the study of partial differential equations in a context which is the least technical possible. As we shall see below, the Littlewood-Paley theory provides a robust approach not only to the separate study of the various regimes of solutions to nonlinear partial differential equations, but also to the fine study of functional inequalities, and how to make them accurate.

\section{The Littlewood-Paley theory: A tool that has become indispensable}

The Littlewood-Paley theory is a localization procedure in the frequency space that, since about three decades ago, has established itself as a very powerful tool in harmonic analysis. The first goal of this text is to present it in a way which is as simple as possible. ${ }^{1}$ Its basic idea is contained in two fundamental inequalities, known as Bernstein's inequalities, that describe some properties of functions whose Fourier transform have compact support.

The first inequality says that, for a tempered distribution ${ }^{2}$ in $\mathbb{R}^{d}$ whose Fourier transform is supported in an annulus of size $\lambda$, to differentiate first and then take the $L^{p}$ norm is the same as to apply a homothety of ratio $\lambda$ on the $L^{p}$ norm.

1 For a more detailed presentation of this theory the reader can consult the monograph [3].

2 A tempered distribution is an element of the topological dual of the Schwartz space $\mathcal{S}\left(\mathbb{R}^{d}\right)$.
In the $L^{2}$ setting this remarkable property is an easy consequence of the action of the Fourier transform on derivatives and of the Fourier-Plancherel formula. The proof in the case of general $L^{p}$ spaces uses Young's inequalities and the fact that the Fourier transform of a convolution is the product of the Fourier transforms.

On the other hand, the second inequality tells us that, for such a distribution, the change from the $L^{p}$ norm to the $L^{q}$ norm, with $q \geq p \geq 1$, costs $\lambda^{d\left(\frac{1}{p}-\frac{1}{q}\right)}$, which must be understood as a Sobolev embedding. It is proved, like the first inequality, using Young's inequalities and the relation between the Fourier transform and the convolution product.

Fourier analysis is at the heart of the Littlewood-Paley theory, which has inspired a large number of my works. It was in conducting experiments on the propagation of heat at the end of the 18th century that Joseph Fourier opened the door to that theory, which was hugely expanded in the 20th century and intervenes in the majority of branches of physics.

In this theory, which bears the name of its creator, one performs the frequency analyis of a function $f$ of $L^{1}\left(\mathbb{R}^{d}\right)$ by the formula:

$$
\widehat{f}(\xi)=\int_{\mathbb{R}^{d}} \mathrm{e}^{-i x \cdot \xi} f(x) d x
$$

Under appropriate conditions, $\widehat{f}$ the Fourier transform of $f$ (also denoted $\mathcal{F} f$ in the present text), allows the synthesis of $f$ through the inversion formula:

$$
f(x)=\frac{1}{(2 \pi)^{d}} \int_{\mathbb{R}^{d}} \mathrm{e}^{i x \cdot \xi} \widehat{f}(\xi) d \xi .
$$


As a consequence, we obtain the Fourier-Plancherel identity

$$
\int_{\mathbb{R}^{d}}|f(x)|^{2} d x=\frac{1}{(2 \pi)^{d}} \int_{\mathbb{R}^{d}}|\widehat{f}(\xi)|^{2} d \xi .
$$

In fact, for all functions $f$ of $\mathcal{S}\left(\mathbb{R}^{d}\right)$, we have, due to Fubini's theorem,

$$
\begin{aligned}
\int_{\mathbb{R}^{d}} f(x) \bar{f}(x) d x & =\frac{1}{(2 \pi)^{d}} \int_{\mathbb{R}^{d}}\left(\int_{\mathbb{R}^{d}} \mathrm{e}^{i x \cdot \xi} \widehat{f}(\xi) d \xi\right) \bar{f}(x) d x \\
& =\frac{1}{(2 \pi)^{d}} \int_{\mathbb{R}^{d}} \widehat{f}(\xi) \overline{\left(\int_{\mathbb{R}^{d}} \mathrm{e}^{-i x \cdot \xi} f(x) d x\right)} d \xi .
\end{aligned}
$$

This representation created a true revolution in the way we think about functions. To give $\widehat{f}$ is exactly equivalent to giving $f$, and this duality between analysis in amplitude (in the physical space described by $x$ ) and analysis in frequency (in the frequency space described by $\xi$ ) is of extraordinary importance in physics and in mathematics.

A fundamental fact from the theory of distributions is that the Fourier transform can be extended to the space of tempered distributions $\mathcal{S}^{\prime}\left(\mathbb{R}^{d}\right)$. The crucial point is the fact that $\mathcal{F}$ is a well-known isomorphism on the Schwartz space $\mathcal{S}\left(\mathbb{R}^{d}\right)$ (the space of smooth functions that, together with all their derivatives, decrease faster than every polynomial) and its extension to $\mathcal{S}^{\prime}\left(\mathbb{R}^{d}\right)$ is defined by duality. ${ }^{3}$

Fourier transforms have a very large number of properties that we do not wish to list here. Let us just recall the two basic principles of these transforms that we cannot dissociate from the convolution product. The first principle of the Fourier transform is that regularity implies decreasing; the second one is that decreasing leads to regularity. The usefulness of these properties, that play a crucial role in the study of Fourier transforms in $\mathcal{S}\left(\mathbb{R}^{d}\right)$, will quickly become clear in what follows.

Fourier analysis allows us to explicitly solve linear equations with constant coefficients. ${ }^{4}$ In particular, by combining the Fourier transform with the convolution product we can explicitly determine the solutions of the Schrödinger equation, a fundamental equation in quantum mechanics:

$$
\text { (S) }\left\{\begin{array}{l}
i \partial_{t} v+\Delta v=0 \\
v_{\mid t=0}=v_{0} \in \mathcal{S}\left(\mathbb{R}^{d}\right) .
\end{array}\right.
$$

In fact, taking the partial Fourier transform with respect to the variable $x$ we obtain for every $(t, \xi)$ in $\mathbb{R} \times \mathbb{R}^{d}$ :

$$
\left\{\begin{array}{l}
i \partial_{t} \widehat{v}(t, \xi)-|\xi|^{2} \widehat{v}(t, \xi)=0 \\
\widehat{v}(0, \xi)=\widehat{v}_{0}(\xi)
\end{array}\right.
$$

and integrating we get

$$
\widehat{v}(t, \xi)=\mathrm{e}^{-i t|\xi|^{2}} \widehat{v}_{0}(\xi)
$$

Combining the inverse Fourier transform together with the properties of the Fourier transform and the convolution product, we deduce that the solution of (S) for $t \neq 0$ can be written as

$$
v(t, \cdot)=\frac{\mathrm{e}^{i \frac{|x|^{2}}{4 t}}}{(4 \pi i t)^{\frac{d}{2}}} \star v_{0} .
$$

3 For a complete presentation of the theory of distributions we can, for instance, see the fundamental references $[34,36]$.

4 Linear equations with variable coefficients and nonlinear equations require different methods.
By Young's inequality, it follows the fundamental dispersion property

$$
\|v(t, \cdot)\|_{L^{\infty}\left(\mathbb{R}^{d}\right)} \leq \frac{1}{|4 \pi t|^{\frac{d}{2}}}\left\|v_{0}\right\|_{L^{1}\left(\mathbb{R}^{d}\right)} .
$$

This technique of explicit representation of solutions can be adapted to all linear evolution equations with constant coefficients. However, it is not always straightforward to deduce the dispersion effects. In fact, for example, to establish dispersive estimations for the wave equation in $\mathbb{R}^{d}$ requires more elaborate techniques involving oscillating integrals, which necessitate a hypothesis of spectral localization in an annulus of Cauchy data.

The analysis of dispersion, a central problem in linear wave mechanics, provides a framework of formidable effectiveness for solving and analysing nonlinear dispersive partial differential equations. It is thanks to the remarkable work of Robert Strichartz [37] in the late 1970s that we have been able to transcribe dispersion phenomena, which correspond to a pointwise inequality, into robust inequalities. The idea of these estimates, known as Strichartz estimates, is to pass from a pointwise in time decay estimate to a spatial integrability gain after an appropriate time average. These Strichartz estimates, which have experienced a big boom these last few years, go along with the Littlewood-Paley theory: they can be expressed equally in Lebesgue spaces and in Besov spaces which we will define next.

The Littlewood-Paley theory was introduced by John Edensor Littlewood and Raymond Paley $[29,30]$ in the 1930s for the harmonic analysis of $L^{p}$ spaces, but its systematic use in the analysis of partial differential equations is more recent. In fact, the main breakthrough of this theory was made after the seminal paper [12] by Jean-Michel Bony in 1981 on the paradifferential calculus that connects nonlinear functions and the Littlewood-Paley decomposition.

The main idea of this theory consists in sampling the frequencies by means of a decomposition of the frequency space in annulus of size $2^{j}$, thus allowing the decomposition of a function into a sum of a countable number of regular functions whose Fourier transform is supported in an annulus of size $2^{j}$ :

$$
f=\sum_{j \in \mathbb{Z}} \dot{\Delta}_{j} f,
$$

where the homogeneous dyadic blocks of $f, \dot{\Delta}_{j} f$, are defined by the filtering of $f$ at frequencies of order $2^{j}$. Observe that this so-called homogeneous Littlewood-Paley decomposition is valid modulo polynomials $P$. In fact, since the Fourier transform of every polynomial is supported at the origin, the identity (1) cannot be applied to polynomials. This restriction on the lower frequencies is overcome in the case of the inhomogeneous Littlewood-Paley decomposition:

$$
f=\sum_{j \geq-1} \Delta_{j} f
$$

where $\Delta_{j} f:=\dot{\Delta}_{j} f$ for $j$ varying in $\mathbb{N}$ and $\Delta_{-1} f$ is an operator filtering the lower frequencies, that is: it only preserves the frequencies in a ball centered at the origin.

The Littlewood-Paley decompositions (1) and (2) introduced above are obtained by a decomposition in the space of frequencies arising from dyadic partitions of unity. More 
precisely, if we are given a radial function $\chi$ belonging to $\mathcal{D}(B(0,4 / 3))$, identically equal to 1 in $B(0,3 / 4)$, we have the following identities

$\chi+\sum_{j \geq 0} \varphi\left(2^{-j} \cdot\right)=1$ in $\mathbb{R}^{d}$, and $\sum_{j \in \mathbb{Z}} \varphi\left(2^{-j} \cdot\right)=1$ in $\mathbb{R}^{d} \backslash\{0\}$, where $\varphi$ is the function defined by $\varphi(\xi)=\chi(\xi / 2)-\chi(\xi)$.

With this normalization $\varphi$ is a radial function of $\mathcal{D}(C)$ where $C$ is the annulus centered at the origin with inner radius $3 / 4$ and outer radius $8 / 3$ and we define the homogeneous dyadic blocks $\dot{\Delta}_{j}$ by ${ }^{5}$

$$
\begin{array}{r}
\dot{\Delta}_{j} f:=\varphi\left(2^{-j} D\right) f:=\mathcal{F}^{-1}\left(\varphi\left(2^{-j} \cdot\right) \mathcal{F} f\right)= \\
2^{j d} h\left(2^{j} \cdot\right) \star f \\
\text { with } h=\mathcal{F}^{-1} \varphi
\end{array}
$$

and the inhomogeneous dyadic blocks $\Delta_{j}$ by $\Delta_{j} f:=\dot{\Delta}_{j} f=$ $2^{j d} h\left(2^{j} \cdot\right) \star f$ if $j \geq 0$ and

$$
\Delta_{-1} f:=\chi(D) f:=\mathcal{F}^{-1}(\chi \mathcal{F} f)=\widetilde{h} \star f \text {, where } \widetilde{h}=\mathcal{F}^{-1} \chi \text {. }
$$

In a similar way, we also introduce the low-frequency cut-off operators

$$
\begin{array}{r}
\dot{S}_{j} f:=\sum_{k \leq j-1} \dot{\Delta}_{k} f:=\mathcal{F}^{-1}\left(\chi\left(2^{-j} \cdot\right) \mathcal{F} f\right)=2^{j d} \widetilde{h}\left(2^{j} \cdot\right) \star f \\
\text { for } j \in
\end{array}
$$

and

$$
S_{j} f:=\sum_{k \leq j-1} \Delta_{k} f=2^{j d} \widetilde{h}\left(2^{j} \cdot\right) \star f \text { for } j \in \mathbb{N} .
$$

It is worth noting that the dyadic blocks that are frequency cut-off operators are convolution operators. This property, which is a trivial consequence of the fact that the Fourier transform changes the convolution product to the pointwise product of functions, plays a central role in the techniques arising from Littlewood-Paley theory. In particular, all of these operators act in the spaces $L^{p}$ in a uniform way with respect to $p$ and $j$.

In what follows, it is also important to underline that the properties of the supports of the functions $\varphi$ and $\chi$ give rise to quasi-orthogonal relations for the Littlewood-Paley decomposition, namely

$$
\dot{\Delta}_{j} \dot{\Delta}_{k}=0 \text { and } \Delta_{j} \Delta_{k}=0 \text { if }|j-k|>1,
$$

which easily implies that

$$
\forall \xi \in \mathbb{R}^{d}, \frac{1}{2} \leq \chi^{2}(\xi)+\sum_{j \geq 0} \varphi^{2}\left(2^{-j} \xi\right) \leq 1,
$$

and

$$
\forall \xi \in \mathbb{R}^{d} \backslash\{0\}, \frac{1}{2} \leq \sum_{j \in \mathbb{Z}} \varphi^{2}\left(2^{-j} \xi\right) \leq 1 .
$$

Littlewood-Paley analysis allows the exact characterization of the regularity of a function $f$ in terms of the decay properties of its dyadic blocks with respect the summation index $j$. We thus recover, in a more precise way, the idea already present in Fourier analysis: that space regularity is translated into frequency decay.

5 By $\mathcal{F}^{-1}$ we denote the inverse Fourier transform in $\mathbb{R}^{d}$ and $\mathcal{F}\left(\varphi\left(2^{-j} D\right) f\right)(\xi)=\varphi\left(2^{-j} \xi\right) \widehat{f}(\xi)$ which shows that $\mathcal{F}\left(\dot{\Delta}_{j} f\right)$ is supported in the annulus $2^{j} \mathrm{C}$.
In particular, using the Fourier-Plancherel formula and the quasi-orthogonality properties (3)-(4), it is easy to observe that we can characterize a function $f$ as an element of $L^{2}\left(\mathbb{R}^{d}\right)$ in terms of the sequence $\left(\left\|\dot{\Delta}_{j} f\right\|_{L^{2}\left(\mathbb{R}^{d}\right)}\right)_{j \in \mathbb{Z}}$ in $\ell^{2}(\mathbb{Z})$, and the same for its inhomogeneous dyadic blocks. More precisely, thanks to an elementary Hilbertian analysis lemma, we can show the existence of a constant $C$ such that we have

$$
C^{-1} \sum_{j \in \mathbb{Z}}\left\|\dot{\Delta}_{j} f\right\|_{L^{2}\left(\mathbb{R}^{d}\right)}^{2} \leq\|f\|_{L^{2}\left(\mathbb{R}^{d}\right)}^{2} \leq C \sum_{j \in \mathbb{Z}}\left\|\dot{\Delta}_{j} f\right\|_{L^{2}\left(\mathbb{R}^{d}\right)}^{2},
$$

and

$$
C^{-1} \sum_{j \geq-1}\left\|\Delta_{j} f\right\|_{L^{2}\left(\mathbb{R}^{d}\right)}^{2} \leq\|f\|_{L^{2}\left(\mathbb{R}^{d}\right)}^{2} \leq C \sum_{j \geq-1}\left\|\Delta_{j} f\right\|_{L^{2}\left(\mathbb{R}^{d}\right)}^{2} .
$$

Similarly, several classic norms can be written in terms of the Littlewood-Paley decomposition. This is, for example, the case of the Sobolev and Hölder norms. In particular, the fact that some function belongs to some Sobolev (resp. Hölder) space is related with properties of decay with respect to $j$ of the $L^{2}$ (resp. $L^{\infty}$ ) norm of $\dot{\Delta}_{j} u$ or $\Delta_{j} u$, according to whether they are homogeneous or nonhomogeneous spaces.

Let us recall that the nonhomogeneous Sobolev spaces $H^{s}\left(\mathbb{R}^{d}\right)$ that naturally show up in a large number of mathematical physics problems are, in the case when $s=m \in \mathbb{N}$, the subspaces of functions $f$ of $L^{2}\left(\mathbb{R}^{d}\right)$ for which all derivatives (in the sense of distributions) of order smaller than or equal to $m$ belong to $L^{2}\left(\mathbb{R}^{d}\right)$. It is then clear, given the quasiorthogonality of the Littlewood-Paley decomposition and the action of the Fourier transform on the derivatives, that the fact that a function is in $H^{m}\left(\mathbb{R}^{d}\right)$ is characterized as follows:

$$
\|f\|_{H^{m}\left(\mathbb{R}^{d}\right)} \sim\left\|\left(2^{j m}\left\|\Delta_{j} f\right\|_{L^{2}\left(\mathbb{R}^{d}\right)}\right)\right\|_{\ell^{2}(j \geq-1)} .
$$

A similar equivalence holds in the case of homogeneous Sobolev spaces $\dot{H}^{m}\left(\mathbb{R}^{d}\right)$, which are more appropriate to study scale invariant problems such as the incomprehensible Navier-Stokes system ${ }^{6}$ and several variants of this system in meteorology and oceanography, or nonlinear wave equations that we have studied in $[1,2,7]$, and many other equations such as those dealt with in $[25,26]$.

In general, to say that a function $f$ belongs to $H^{s}\left(\mathbb{R}^{d}\right)$ means, roughly speaking, that $f$ has $s$ derivatives (fractional derivatives if $s$ is noninteger) in $L^{2}\left(\mathbb{R}^{d}\right)$, and, as before, we can prove that there exists a constant $C$ such that

$$
\begin{aligned}
C^{-1} \sum_{j \geq-1} 2^{2 j s}\left\|\Delta_{j} f\right\|_{L^{2}\left(\mathbb{R}^{d}\right)}^{2} & \leq\|f\|_{H^{s}\left(\mathbb{R}^{d}\right)}^{2} \\
& \leq C \sum_{j \geq-1} 2^{2 j s}\left\|\Delta_{j} f\right\|_{L^{2}\left(\mathbb{R}^{d}\right)}^{2} .
\end{aligned}
$$

This heuristic idea can also be applied to the homogeneous Sobolev norms, giving rise to the following correspondence in the setting of the Littlewood-Paley theory:

$$
\begin{aligned}
C^{-1} \sum_{j \in \mathbb{Z}} 2^{2 j s}\left\|\dot{\Delta}_{j} f\right\|_{L^{2}\left(\mathbb{R}^{d}\right)}^{2} & \leq\|f\|_{\dot{H}^{s}\left(\mathbb{R}^{d}\right)}^{2} \\
& \leq C \sum_{j \in \mathbb{Z}} 2^{2 j s}\left\|\dot{\Delta}_{j} f\right\|_{L^{2}\left(\mathbb{R}^{d}\right)}^{2} .
\end{aligned}
$$

6 Recall that for the incomprehensible Navier-Stokes system the question of eventual creation of singularities after a finite time is one of the Millenium problems proposed by the Clay Mathematics Institute. 
In examining these inequalities we observe that three parameters play a role: the regularity parameter $s$, the exponent of the Lebesgue norm used to measure the dyadic blocks $\dot{\Delta}_{j} f$ or $\Delta_{j} f$ and the type of sum preformed, either over $\mathbb{Z}$ or for $j \geq-1$. This observation allows, more generally, to efficiently characterise the norms of homogeneous or nonhomogeneous Besov spaces, respectively $\dot{B}_{p, r}^{s}\left(\mathbb{R}^{d}\right)$ and $B_{p, r}^{s}\left(\mathbb{R}^{d}\right)$. The norms of these spaces, which can be defined in terms of finite differences or using the heat kernel (as we can see, for example, in $[3,40]$ ) can be expressed in terms of Littlewood-Paley decompositions ${ }^{7}$ :

$$
\|f\|_{B_{p, r}^{s}\left(\mathbb{R}^{d}\right)} \sim\left(\sum_{j \geq-1} 2^{r j s}\left\|\Delta_{j} f\right\|_{L^{p}\left(\mathbb{R}^{d}\right)}^{r}\right)^{\frac{1}{r}},
$$

and

$$
\|f\|_{\dot{B}_{p, r}^{s}\left(\mathbb{R}^{d}\right)} \sim\left(\sum_{j \in \mathbb{Z}} 2^{r j s}\left\|\dot{\Delta}_{j} f\right\|_{L^{p}\left(\mathbb{R}^{d}\right)}^{r}\right)^{\frac{1}{r}} .
$$

Even if scale invariant, the homogeneous Sobolev spaces (and more generally the homogeneous Besov spaces) have to be manipulated with care, since, as was mentioned above, the homogeneous Littlewood-Paley decomposition (1) is only defined as modulo polynomials of arbitrary degree. There is no consensus about the definition of these spaces. In certain references, such as [11], they are defined as modulo polynomials of arbitrary degree. In others, such as [3], they are defined subject to a condition on the low frequencies. This condition requires limiting oneself to tempered distributions $f$ satisfying (in the sense of distributions)

$$
\left\|\dot{S}_{j} f\right\|_{L^{\infty}\left(\mathbb{R}^{d}\right)} \stackrel{j \rightarrow-\infty}{\longrightarrow} 0 .
$$

The dyadic decompositions provide not only the possibility of characterising a function as an element of almost all the classical spaces (Hölder, Sobolev, Besov, Lebesgue, TriebelLizorkin) by conditions concerning only its dyadic blocks, but they also allow us to define a plethora of functional spaces.

Littlewood-Paley decompositions and more simply the decomposition of functions into low and high frequency components are techniques that have proved their usefulness in the study of functional inequalities and in the analysis of nonlinear partial differential equations.

Sobolev embeddings are among the most celebrated of all functional inequalities. They provide key tools for the study of linear and nonlinear partial differential equations, in the elliptic, parabolic or hyperbolic framework. Sobolev inequalities express a strong integrability or regularity property for a function $f$ in terms of integrability properties of some derivatives of $f$.

Among those inequalities, we can mention the Sobolev inequalities in Lebesgue spaces:

$$
\dot{H}^{s}\left(\mathbb{R}^{d}\right) \hookrightarrow L^{p}\left(\mathbb{R}^{d}\right),
$$

with $0 \leq s<d / 2$ and $p=2 d /(d-2 s)$.

Let us observe that the value $p=2 d /(d-2 s)$ can easily be deduced using an homogeneity argument. In fact, if for every function $v$ defined in $\mathbb{R}^{d}$ and all $\lambda>0$ we define a function $v_{\lambda}$ by $v_{\lambda}(x)=v(\lambda x)$, it is easy to verify that

$$
\left\|v_{\lambda}\right\|_{L^{p}\left(\mathbb{R}^{d}\right)}=\lambda^{-\frac{d}{p}} \quad \text { and } \quad\left\|v_{\lambda}\right\|_{\dot{H}^{s}\left(\mathbb{R}^{d}\right)}=\lambda^{s-\frac{d}{2}}\left\|v_{\lambda}\right\|_{\dot{H}^{s}\left(\mathbb{R}^{d}\right)} .
$$

7 Observe that the Besov spaces are independent of the dyadic blocks $\dot{\Delta}_{j}$ and $\Delta_{j}$.
Since both quantities $\|\cdot\|_{L^{p}\left(\mathbb{R}^{d}\right)}$ and $\|\cdot\|_{\dot{H}^{s}\left(\mathbb{R}^{d}\right)}$ have the same homogeneity degree when the Lebesgue index $p=2 d /(d-$ $2 s$ ) (which means that they behave in the same way under a change of the unit of length), it is thus natural to compare them and we can assume in what follows that $\|f\|_{\dot{H}^{s}\left(\mathbb{R}^{d}\right)}=1$.

We know that for all real number $p \geq 1$ and all measurable function $f$, we have, due to Fubini's theorem,

$$
\|f\|_{L^{p}\left(\mathbb{R}^{d}\right)}^{p}=p \int_{0}^{\infty} \lambda^{p-1} \mu(|f|>\lambda) d \lambda .
$$

To establish the Sobolev embedding (5), we decompose $f$ into low and high frequency components in the following way:

$$
f=f_{\ell, A}+f_{h, A} \quad \text { with } \quad f_{\ell, A}=\mathcal{F}^{-1}\left(\mathbf{1}_{B(0, A)} \widehat{f}\right) .
$$

Since the support of the Fourier transform of $f_{\ell, A}$ is a compact set, the function $f_{\ell, A}$ is bounded and, more precisely, by using the inversion formula and the Cauchy-Schwarz inequality, we have

$$
\begin{aligned}
\left\|f_{\ell, A}\right\|_{L^{\infty}\left(\mathbb{R}^{d}\right)} & \leq(2 \pi)^{-d}\left\|\widehat{f_{\ell, A}}\right\|_{L^{1}\left(\mathbb{R}^{d}\right)} \\
& \leq(2 \pi)^{-d} \int_{\mathbb{R}^{d}}|\xi|^{s}|\xi|^{-s}\left|\widehat{f_{\ell, A}}(\xi)\right| d \xi \\
& \leq C_{s} A^{\frac{d}{2}-s}\|f\|_{\dot{H}^{s}\left(\mathbb{R}^{d}\right)} .
\end{aligned}
$$

Now, the triangle inequality implies, for all $A>0$,

$$
(|f|>\lambda) \subset\left(\left|f_{\ell, A}\right|>\lambda / 2\right) \cup\left(\left|f_{h, A}\right|>\lambda / 2\right) .
$$

Consequently, by choosing

$$
A=A_{\lambda} \stackrel{\text { def }}{=}\left(\frac{\lambda}{4 C_{s}}\right)^{\frac{p}{d}},
$$

we deduce that

$$
\|f\|_{L^{p}\left(\mathbb{R}^{d}\right)}^{p} \leq p \int_{0}^{\infty} \lambda^{p-1} \mu\left(\left|f_{h, A_{\lambda}}\right|>\lambda / 2\right) d \lambda .
$$

Since, by the Bienaymé-Tchebychev inequality,

$$
\mu\left(\left|f_{h, A_{\lambda}}\right|>\lambda / 2\right) \leq 4 \frac{\left\|f_{h, A_{\lambda}}\right\|_{L^{2}\left(\mathbb{R}^{d}\right)}^{2}}{\lambda^{2}},
$$

we obtain

$$
\|f\|_{L^{p}\left(\mathbb{R}^{d}\right)}^{p} \leq 4 p \int_{0}^{\infty} \lambda^{p-3}\left\|f_{h, A_{\lambda}}\right\|_{L^{2}\left(\mathbb{R}^{d}\right)}^{2} d \lambda .
$$

Finally, by the Fourier-Plancherel identity,

$$
\left\|f_{h, A_{\lambda}}\right\|_{L^{2}\left(\mathbb{R}^{d}\right)}^{2}=(2 \pi)^{-d} \int_{\left(|\xi| \geq A_{\lambda}\right)}|\widehat{f}(\xi)|^{2} d \xi,
$$

which implies, due to Fubini's theorem, that for all $p>2$

$$
\begin{aligned}
\|f\|_{L^{p}\left(\mathbb{R}^{d}\right)}^{p} & \leq 4 p(2 \pi)^{-d} \int_{\mathbb{R}^{d}}\left(\int_{0}^{4 C_{s}|\xi|^{\frac{d}{p}}} \lambda^{p-3} d \lambda\right)|\widehat{f}(\xi)|^{2} d \xi \\
& \leq C_{p} \int_{\mathbb{R}^{d}}|\xi|^{\frac{d(p-2)}{p}}|\widehat{f}(\xi)|^{2} d \xi,
\end{aligned}
$$

where $C_{p}=(2 \pi)^{-d} \frac{4 p}{p-2}\left(4 C_{s}\right)^{p-2}$. Since $s=d\left(\frac{1}{2}-\frac{1}{p}\right)$, this concludes the proof of the Sobolev embedding.

The proof presented above is borrowed from [16]. We have other previous proofs of this estimate, namely one based on the Hardy-Littlewood-Sobolev inequality, which is for instance presented in [3]. We should note that the arguments of the above proof have inspired a number of other works, 
among which we refer to the paper [5] where the authors considered Sobolev embeddings in the Lorentz spaces $L^{p, q}$. Recall that the Lorentz spaces ${ }^{8}$ were introduced in the 1950 s by Lorentz so that $L^{p, \infty}$ are the weak spaces introduced by Marcinkiewicz in the 1930s, and $L^{p, p}$ are the usual Lebesgue spaces $L^{p}$.

This technique of decomposition into low and high frequencies was also relevant for the study of nonlinear partial differential equations, namely to establish that some Cauchy problems are globally well posed. Among these works we can refer to the article of Fujita-Kato [18] on the Navier-Stokes equations. In this type of approach, the idea is to decompose the Cauchy data (assumed here, for simplicity, in some Sobolev space $\dot{H}^{s}$ ) into low and high frequencies in such a way that the high frequency part has rather small norm in $\dot{H}^{s}$. If we have a global existence theorem for small initial data, then this high frequency part will give rise to a global solution to the problem, whereas the low frequency part (that will be regular) will satisfy a modified equation, and all we need to do is to prove that we can solve this perturbed equation.

The Sobolev embedding (5) is invariant by translation and scaling, but it is not invariant by oscillations, that is, by multiplication by oscillating functions, namely by those of the type $u_{\epsilon}(x)=e^{i \frac{(x \mid \omega)}{\epsilon}} \varphi(x)$, where $\omega$ is a unit vector of $\mathbb{R}^{d}$, and $\varphi$ is a function in $\mathcal{S}\left(\mathbb{R}^{d}\right)$. Revisiting the proof of the Sobolev embedding presented above we can establish the following inequality due to Gérard-Meyer-Oru [20] :

$$
\|u\|_{L^{p}\left(\mathbb{R}^{d}\right)} \leq \frac{C}{(p-2)^{\frac{1}{p}}}\|u\|_{\dot{B}_{\infty, \infty}^{s-\frac{d}{2}}\left(\mathbb{R}^{d}\right)}^{1-\frac{2}{p}}\|u\|_{\dot{H}^{s}\left(\mathbb{R}^{d}\right)}^{\frac{2}{p}} .
$$

This Sobolev inequality is sharp, as the oscillatory example $u_{\epsilon}(x)=e^{i \frac{(x \mid \omega)}{\epsilon}} \varphi(x)$ shows. Many other examples show the optimality of the estimate (6), in particular a fractal example constructed in [4], supported in a Cantor type set, and the example of the chirp signal:

$$
f(x)=x^{-\alpha} \sin \left(\frac{1}{x}\right), \alpha>0,
$$

investigated in [5].

The refined estimate (6) is one of the key arguments in [19] where Patrick Gérard gave a characterisation of the defect of compactness of the critical Sobolev embedding (5) by means of profile decompositions. ${ }^{9}$ We recall that the study of the defect of compactness of Sobolev embeddings of functional spaces, which goes back to the seminal works of PierreLouis Lions [27,28], provides a useful tool in the study of geometric problems and the understanding of the behaviour of solutions to nonlinear partial differential equations.

Nonlinear analysis has progressed substantially in the last decades due to profile decomposition techniques. This type of decomposition has been generalised, by different approaches, to other functional settings. In particular, we refer to the recent works [8,9] about the description of the defect of compactness of the critical Sobolev embedding of $H^{1}\left(\mathbb{R}^{2}\right)$ in $\mathcal{L}\left(\mathbb{R}^{2}\right)$, where $\mathcal{L}\left(\mathbb{R}^{2}\right)$, the so-called Orlicz space, ${ }^{10}$ is the space of measur-

8 For more details see $[11,40]$.

9 Profile decompositions originate in the work of Brézis-Coron [15].

10 For an introduction to Orlicz spaces see $[35,41]$. able functions $u: \mathbb{R}^{2} \rightarrow \mathbb{C}$ for which there exists a real number $\lambda>0$, such that

$$
\int_{\mathbb{R}^{2}}\left(e^{\frac{|\mathrm{u}(\mathrm{x})|^{2}}{\lambda^{2}}}-1\right) d x<\infty,
$$

as well as its generalisation to higher dimensions in [10]. This Sobolev embedding, which is based on the TrudingerMoser inequalities, deals with the limiting case of the Sobolev embedding (5) and intervenes in numerous geometrical and physical problems, namely in the propagation of laser beams in different media. The study of this embedding is done in [10] by Fourier analysis arguments that highlight the fact that the elements responsible for the lack of compactness are, in this case and in contradistinction to the case of the Sobolev embedding (5), spread over the frequencies.

It is also noteworthy that an approach started by Stéphane Jaffard in [23] has allowed the extension of Patrick Gérard's result in [19] to the setting of the Triebel-Lizorkin spaces and has inspired the abstract analysis in [6]. This approach was based on the theory of wavelets, which, for its part, was inspired by the Littlewood-Paley, and will be discussed later.

As was referred to above, the second Bernstein inequality must be understood as a Sobolev embedding. In fact, it is easy to deduce from this second inequality that for all real numbers $s$, and for all $1 \leq p_{1} \leq p_{2} \leq \infty$ and $1 \leq r_{1} \leq r_{2} \leq \infty$ we have

$$
\dot{B}_{p_{1}, r_{1}}^{s}\left(\mathbb{R}^{d}\right) \hookrightarrow \dot{B}_{p_{2}, r_{2}}^{s-d\left(\frac{1}{p_{1}}-\frac{1}{p_{2}}\right)}\left(\mathbb{R}^{d}\right),
$$

and analogously for the nonhomogeneous case.

Observe that these Sobolev embeddings are strict, as is shown, in the particular case of the Sobolev embedding $\dot{H}^{s}\left(\mathbb{R}^{d}\right) \hookrightarrow \dot{B}_{2, \infty}^{s}\left(\mathbb{R}^{d}\right)$, by the following example based on the idea of lacunar series. Given a function $\chi$ of $\mathcal{S}\left(\mathbb{R}^{d}\right)$ whose Fourier transform is supported in a small ball centered at 0 with radius $\epsilon_{0}$, and given a vector $\omega \in \mathbb{R}^{d}$ with Euclidean norm $3 / 2$, we consider the sequence of functions $\left(f_{n}\right)_{n \in \mathbb{N}}$ defined by

$$
f_{n}(x)=\sqrt{n} \sum_{j \geq n} 2^{-j s} \frac{1}{j+1} e^{i 2^{j}(x \mid \omega)} \chi(x) .
$$

It is easy to observe that

$$
\left\{\begin{array}{l}
\dot{\Delta}_{j} f_{n}=0 \quad \text { if } j \leq n-1 \quad \text { and } \\
\left(\dot{\Delta}_{j} f_{n}\right)(x)=\frac{\sqrt{n} 2^{-j s}}{j+1} e^{i 2^{j}(x \mid \omega)} \chi(x) \text { if } j \geq n .
\end{array}\right.
$$

By an elementary computation, we conclude that

$$
\left\|f_{n}\right\|_{\dot{H}^{s}\left(\mathbb{R}^{d}\right)}^{2} \sim n \sum_{j \geq n} \frac{1}{(j+1)^{2}} \sim 1 \text { and }\left\|f_{n}\right\|_{\dot{B}_{2, \infty}^{s}\left(\mathbb{R}^{d}\right)} \lesssim \frac{1}{\sqrt{n}},
$$

which clearly shows the strict inclusion of $\dot{H}^{s}\left(\mathbb{R}^{d}\right)$ into $\dot{B}_{2, \infty}^{s}\left(\mathbb{R}^{d}\right)$.

The techniques arising from the Littlewood-Paley theory allow also the analysis of the product of two tempered distributions (if it exists) by means of J.-M. Bony's paradifferential calculus. It does so in the following way: given two tempered distributions $u$ and $v$, we write

$$
u=\sum_{p} \Delta_{p} u \text { and } v=\sum_{q} \Delta_{q} v .
$$

Formally, if the product exists it is written as

$$
u v=\sum_{p, q} \Delta_{p} u \Delta_{q} v .
$$


The idea consists of decomposing the product $u v$ into three parts: a first one with terms where the frequencies of $u$ are large compared with those of $v$, a second one with terms where the frequencies of $v$ are large compared with those of $u$ and a third one for which the frequencies of $u$ and $v$ have comparable sizes. This leads to the following definition, first introduced by Jean-Michel Bony in [12]: we write

$$
\begin{aligned}
u v & =T_{u} v+T_{v} u+R(u, v) \quad \text { with } \\
T_{u} v & \stackrel{\text { def }}{=} \sum_{p \leq q-2} \Delta_{p} u \Delta_{q} v=\sum_{q} S_{q-1} u \Delta_{q} v \\
R(u, v) & \stackrel{\text { def }}{=} \sum_{|q-p| \leq 1} \Delta_{q} u \Delta_{p} v .
\end{aligned}
$$

This so-called Jean-Michel Bony's decomposition is fundamental to the study of product laws as well as to the study of nonlinear partial differential equations. Clearly, it admits a homogeneous version. Let us recall that the bilinear operator $T_{u} v$ is called the paraproduct of $v$ by $u$, whereas the symmetric bilinear operator $R(u, v)$ is called the remainder.

From the detailed study of the way the paraproduct and the remainder act on Sobolev, Hölder, and, more generally, Besov spaces, one can identify some principles:

- For two compactly supported distributions, the paraproduct is always defined, and the regularity of $T_{u} v$ is determined, mainly, by the regularity of $v$.

- On the other hand, the remainder is not always defined, but when it is the regularities of $u$ and $v$ add up to determine its regularity.

Jean-Michel Bony's paradifferential calculus has proven to be very effective in the study of evolution equations, which describe the behaviour of a physical phenomenon dependent of time. This method's relevance will be illustrated by presenting a method of microlocal decomposition we have introduced in $[1,2]$ in collaboration with Jean-Yves Chemin (see also $[38,39])$ for the study of quasilinear wave equations of the type

$$
(E)\left\{\begin{aligned}
\partial_{t}^{2} u-\Delta u-\partial(G(u) \partial u) & =Q(\nabla u, \nabla u) \\
\left(u, \partial_{t} u\right)_{\mid t=0} & =\left(u_{0}, u_{1}\right)
\end{aligned}\right.
$$

with

$$
\partial(G \partial u)=\sum_{1 \leq j, k \leq d} \partial_{j}\left(G^{j, k} \partial_{k} u\right),
$$

where $Q$ is a quadratic form on $\mathbb{R}^{1+d}$; and $G$ is a $C^{\infty}$ function vanishing on 0 , which, together with all its derivatives is bounded from $\mathbb{R}$ into the space of symmetric matrices on $\mathbb{R}^{d}$, and takes its values in a compact set $K$ such that $I d+K$ is included in the cone of symmetric positive definite matrices.

By the classical theory of strictly hyperbolic equations, ${ }^{11}$ we can solve such equations with Cauchy data $\left(u_{0}, u_{1}\right)$ in the space $\dot{H}^{s}\left(\mathbb{R}^{d}\right) \times \dot{H}^{s-1}\left(\mathbb{R}^{d}\right)$ for $s>\frac{d}{2}+1$. Notwithstanding, it is important to think about the scale invariance of such equations. It can be checked immediately that if $u$ is a solution of equation $(E)$, then the function $u_{\lambda}$ defined by $u_{\lambda}(t, x)=$ $u(\lambda t, \lambda x)$ is also a solution of $(E)$. A large number of works have been concerned in solving nonlinear wave equations by trying to decrease as far as possible the index of minimal

11 See, for instance, Chapter 4 of [3]. regularity of the initial data towards a space of initial data invariant by the above change of scale, for instance in the space $\dot{H}^{\frac{d}{2}}$.

The goal here is to solve equation $(E)$ for less regular Cauchy data than what is required by energy methods. This approach fits in Christodoulou-Klainerman programme for general relativity, which also includes works by Klainerman, Bourgain, Tao and their schools. To get closer to scale invariant spaces for the initial data, it is obvious that we need to use the specific properties of the wave equation, namely the dispersion effects referred to above. This necessitates the proof of Strichartz type inequalities for that equation that we can interpret as a wave equation with variable and rough coefficients. It is the alliance of geometric optics and harmonic analysis through the paradifferential calculus of Jean-Michel Bony that allows us to establish these estimates, to improve the minimal regularity index and to give an answer to a longstanding open question.

As stated above, Strichartz estimates are obtained from dispersive phenomena coupled with an abstract functional argument known as $T T^{*}$-argument, developed by Ginibre and Velo in [21], and generalised by Keel and Tao in [24]. As also pointed out previously, dispersive phenomena are obtained for the wave equations with constant coefficients by applying a stationary phase argument to an explicit representation of the solution. The variable coefficients case requires more attention, since in this case we do not have an explicit representation, and we recur to geometric optics methods involving Hamilton-Jacobi and transport equations to approximate the solution. When the coefficients are rough, as, for example, in the quasilinear case, such an approach does not work, since the Hamilton-Jacobi equation produces singularities. It is the Littlewood-Paley theory that allow us to overcome this difficulty.

In fact, to perform such a method in this framework requires a regularisation of the coefficients. More precisely, using Bony's paradifferential calculus, we are left with the study of the part of the solution related to frequencies of size $2^{j}$, which satisfies a wave equation with regular coefficients. By a classical method, we construct a microlocal approximation of the solution to this equation, that is valid in a time interval whose size depends on the frequency and that allows us to establish a microlocal Strichartz estimate. In fact, it seems impossible to construct a local approximation of the solution since the associated Hamilton-Jacobi equation generates singularities at a time related to the frequency: this is due to the fact that these regular coefficients keep memory of the original regularity of the solution. The local Strichartz estimate is obtained (with some loss) by decomposing the interval $[0, T]$ into intervals where the microlocal Strichartz estimate is satisfied.

The applications of the Littlewood-Paley theory, and particularly of the paradifferential calculus, are manifold and we cannot enumerate all of them here. For a wider range of perspectives, whether in the study of functional inequalities or the analysis of solutions to nonlinear partial differential equations arising in fluid mechanics or general relativity, we refer the reader to the monograph [3].

The Littlewood-Paley theory has inspired the wavelet theory, which is at the origin of numerous progresses in various 
applied disciplines, such as signal and image processing techniques. We can illustrate wavelet theory in a simple setting by considering Haar's system introduced at the beginning of the 1920s by Alfred Haar in his PhD thesis. This system is defined by the functions

$$
\psi_{j, k}(x)=2^{\frac{j}{2}} \psi\left(2^{j} x-k\right), \quad j, k \in \mathbb{Z},
$$

where the generating wavelet

$$
\psi=\chi_{\left[0, \frac{1}{2}[\right.}-\chi_{\left[\frac{1}{2}, 1\right]}
$$

is the piecewise constant function equal to 1 in $\left[0, \frac{1}{2}[\right.$ and -1 in $[1 / 2,1[$. This system constitutes an orthonormal basis of $L^{2}(\mathbb{R})$ and, thus, it is straightforward that all functions $f$ of $L^{2}(\mathbb{R})$ can be decomposed as follows:

$$
f=\sum_{j, k \in \mathbb{Z}}\left\langle f, \psi_{j, k}\right\rangle \psi_{j, k},
$$

where $\left\langle f, \psi_{j, k}\right\rangle$ denotes the scalar product of $f$ and $\psi_{j, k}$ in $L^{2}(\mathbb{R})$. In the wavelet decomposition (8), the homogeneous dyadic blocs $\dot{\Delta}_{j} f$ are replaced by the projections

$$
P_{j} f=\sum_{k \in \mathbb{Z}}\left\langle f, \psi_{j, k}\right\rangle \psi_{j, k},
$$

where the index $k$ provides an additional level of discretisation.

The main drawback of Haar's system is its lack of regularity, since the mother wavelet $\psi$ is not continuous. Other more regular wavelet bases were constructed later on, allowing us to get decompositions in wavelets similar to (8), often taking into consideration the scaling of the space in question.

As in the Littlewood-Paley decompositions, we can characterise the belonging of a function to almost all classical functional spaces by conditions pertaining only to the absolute values of the coefficients of the function in a basis of unconditional normalised wavelets. ${ }^{12}$

For example, in the Besov space $\dot{B}_{p, p}^{s}\left(\mathbb{R}^{d}\right), 1 \leq p<\infty$ and $s<\frac{d}{p}$, the wavelet decomposition of a function takes the form:

$$
f=\sum_{\lambda \in \nabla} d_{\lambda} \psi_{\lambda}
$$

where $\lambda=(j, k)$ includes the scale index $j=j(\lambda)$ and the space index $k=k(\lambda)$, and

$$
\psi_{\lambda}=\psi_{j, k}=2^{j r} \psi\left(2^{j} \cdot-k\right), \quad j \in \mathbb{Z}, k \in \mathbb{Z}^{d},
$$

where $\psi$ is the mother wavelet, and $r=\frac{d}{p}-s \cdot$ The wavelet theory allows to characterise the belonging to $\dot{B}_{p, p}^{s}\left(\mathbb{R}^{d}\right)$ in terms of the coefficients in the above wavelet decomposition as follows:

$$
\|f\|_{\dot{B}_{p, p}^{s}\left(\mathbb{R}^{d}\right)} \sim\left\|\left(d_{\lambda}\right)_{\lambda \in \nabla}\right\|_{\ell^{p}} .
$$

The possibility of characterising the regularity of a function by the size of its wavelet coefficients is at the heart of the extensive applications of wavelet theory. In particular, we can translate the equivalence (10) by the decrease of the wavelet coefficients, with the exception of a small number of them. This property of concentration of information in a small number of coefficients, often called parsimony or sparsity, plays a crucial role in image processing. In this type of essentially

12 For more details consult $[17,32,33]$ nonlinear process, it is clear that the set of remaining coefficients depends on the function we are approaching. A general theory for the study of these phenomena, known as nonlinear approximation theory, was started by Ronald DeVore in the 1980s.

A first result in nonlinear approximation theory is the representation of a function by its $N$ most significant coefficients. More precisely, given an element $f$ of $\dot{B}_{p, p}^{s}\left(\mathbb{R}^{d}\right)$ admitting a decomposition given by (9) in the wavelet basis $\left(\psi_{\lambda}\right)_{\lambda \in \nabla}$, the goal is to keep only the nonlinear projection $Q_{N} f$ defined by

$$
Q_{N} f=\sum_{\lambda \in E_{N}} d_{\lambda} \psi_{\lambda},
$$

where $E_{N}=E_{N}(f)$ is the subset of $\nabla$ with cardinal $N$, which corresponds to the $N$ largest wavelet coefficients $\left|d_{\lambda}\right|$.

Among the many applications of the nonlinear projection $Q_{N} f$, we can refer to the following estimate:

$$
\sup _{\|f\|_{\dot{B}_{p, p}^{s}\left(\mathbb{R}^{d}\right)} \leq 1}\left\|f-Q_{N} f\right\|_{\dot{B}_{q, q}^{t}\left(\mathbb{R}^{d}\right)} \leq C N^{-\frac{s-t}{d}},
$$

that has played a key role in [6], in the study of the lack of compactness of the critical Sobolev embedding

$$
\dot{B}_{p, p}^{s}\left(\mathbb{R}^{d}\right) \hookrightarrow \dot{B}_{q, q}^{t}\left(\mathbb{R}^{d}\right),
$$

with $0<\frac{1}{p}-\frac{1}{q}=\frac{s-t}{d}$.

In fact, given a function $f$ of $\dot{B}_{p, p}^{s}\left(\mathbb{R}^{d}\right)$ we obtain, from (10) and using $\left(d_{m}\right)_{m>0}$, the decreasing rearrangment of $\left|d_{\lambda}\right|$

$$
\begin{aligned}
\left\|f-Q_{N} f\right\|_{\dot{B}_{q, q}^{t}\left(\mathbb{R}^{d}\right)} & \sim\left(\sum_{\lambda \notin E_{N}}\left|d_{\lambda}\right|^{q}\right)^{1 / q}=\left(\sum_{m>N}\left|d_{m}\right|^{q}\right)^{1 / q} \\
& \leq\left|d_{N}\right|^{1-p / q}\left(\sum_{m>N}\left|d_{m}\right|^{p}\right)^{1 / q} \\
& \leq\left(N^{-1} \sum_{m=1}^{N}\left|d_{m}\right|^{p}\right)^{1 / p-1 / q}\left(\sum_{m>N}\left|d_{m}\right|^{p}\right)^{1 / q} \\
& \leq N^{-(1 / p-1 / q)}\left(\sum_{m>0}\left|d_{m}\right|^{p}\right)^{1 / p} \\
& \leq N^{-\frac{s-t}{d}}\left\|\left(d_{\lambda}\right)_{\lambda \in \nabla}\right\|_{\ell^{p}} \sim N^{-\frac{s-t}{d}}\|f\|_{\dot{B}_{p, p}^{s}\left(\mathbb{R}^{d}\right) .}
\end{aligned}
$$

The success of wavelet theory either in signal and image processing, or in the field of numerical simulations of partial differential equations is now well established. For a general survey of applications of this theory, one can consult the monograph [31] and the references therein.

The Littlewood-Paley theory is considered the simplest tool of microlocal analysis. We can see microlocal analysis as the study of functions by the decomposition of the phase space, that is the space of $(x, \xi)$. In a general way, this process consists of localising in physical space $x$ then in the Fourier variable $\xi$, which corresponds to the localization in a ball for a metric of $T^{\star} \mathbb{R}^{d}$ (the cotangent space of $\mathbb{R}^{d}$ ): it is the WeylHörmander calculus. ${ }^{13}$ The interest in this type of process, introduced in the 1970s, is to allow for the analysis of fine properties of functions defined in the physical space by operating in the phase space, where the number of variables has doubled. This turned out to be particularly useful in the study of

13 See, for example, [13, 14, 22]. 
nonlinear partial differential equations, namely, for instance, to take into consideration certain geometric specificities.

The whole issue of the Weyl-Hörmander calculus consists in the use of reasonable metrics (the so-called Hörmander metrics) in order to localise in phase space. As an example, the procedure of localising in the variable $x$ in an Euclidean ball with size $\alpha$, and afterwards in the Fourier variable in a ball of radius $\alpha\left(1+\left|\xi_{0}\right|^{2}\right)^{\frac{1}{2}}$ is equivalent to localize in a ball for the following metric, the so called $(1,0)$ metric:

$$
g_{(x, \xi)}\left(d x^{2}, d \xi^{2}\right)=d x^{2}+\frac{d \xi^{2}}{1+|\xi|^{2}} .
$$

The so-called Weyl-Hörmander calculus, which achieved its present day formalism at the end of the 1970s in the works of L. Hörmander, generalises this metric. In fact, it consists of the description of reasonable ways to decompose the phase space. These decompositions are chosen according to the nature and the geometry of the problem under consideration. The admissible decompositions are those whose construction is based on Hörmander's metrics, which are functions $g$ of $T^{\star} \mathbb{R}^{d}$ with its standard sympletic structure in the set of positive definite quadratic forms in $T^{\star} \mathbb{R}^{d}$ satisfying:

- a so-called slowness assumption stating that the metric does not change much on its own balls, and this in a uniform way;

- an uncertainty principle hypothesis that prevents too much localisation. In particular, the uncertainty principle imposes that the volume of a $g_{X}$ ball of radius 1 is larger than or equal to the volume of the Euclidean ball of radius 1 ;

- and finally, a so-called temperance hypothesis that reflects the fact that we can estimate the ratio of metrics in arbitrary points by the dual metric.

\section{Bibliography}

[1] H. Bahouri et J.-Y. Chemin, Équations d'ondes quasilinéaires et estimations de Strichartz. American Journal of Mathematics, 121, pages 1337-1377, 1999.

[2] H. Bahouri and J.-Y. Chemin, Microlocal analysis, bilinear estimates and cubic quasilinear wave equation. Astérisque, Bulletin de la Société Mathématique de France, pages 93-142, 2003.

[3] H. Bahouri, J.-Y. Chemin and R. Danchin, Fourier Analysis and Applications to Nonlinear Partial Differential Equations. Grundlehren der Mathematischen Wissenschaften. Springer Verlag, 343, 2011.

[4] H. Bahouri, J.-Y. Chemin and I. Gallagher, Refined Hardy inequalities. Annali della Scuola Normale di Pisa, 59, pages 375-391, 2006.

[5] H. Bahouri and A. Cohen, Refined Sobolev inequalities in Lorentz spaces. Journal of Fourier Analysis and Applications, 17, pages 662-673, 2011.

[6] H. Bahouri, A. Cohen and G. Koch, A general waveletbased profile decomposition in critical embedding of function spaces. Confluentes Mathematici, 3, pages 387-411, 2011.

[7] H. Bahouri and P. Gérard, High frequency approximation of solutions to critical nonlinear wave equations. American Journal of Math, 121, pages 131-175, 1999.

[8] H. Bahouri, M. Majdoub and N. Masmoudi, Lack of compactness in the 2D critical Sobolev embedding. Journal of Functional Analysis, 260, pages 208-252, 2011.

[9] H. Bahouri, M. Majdoub and N. Masmoudi, Lack of compactness in the $2 \mathrm{D}$ critical Sobolev embedding, the general case. Journal de Mathématiques Pures et Appliquées, 101, pages 415-457, 2014.

[10] H. Bahouri and G. Perelman, A Fourier approach to the profile decomposition in Orlicz spaces. Mathematical Research Letters, 21, pages 33-54, 2014.

[11] J. Bergh and J. Löfström, Interpolation Spaces. An Introduction. Springer Verlag, Berlin, 1976.

[12] J.-M. Bony, Calcul symbolique et propagation des singularités pour les équations aux dérivées partielles non linéaires. Annales de l'École Normale Supérieure, 14, pages 209-246, 1981.

[13] J.-M. Bony and J.-Y. Chemin, Espaces fonctionnels associés au calcul de Weyl-Hörmander. Bulletin de la Société Mathématique de France, 122, pages 77-118, 1994.

[14] J.-M. Bony and N. Lerner, Quantification asymptotique et microlocalisation d'ordre supérieur. Annales de l'École Normale Supérieure, 22, pages 377-433, 1989.

[15] H. Brézis and J.-M. Coron, Convergence of solutions of HSystems or how to blow bubbles. Archive for Rational Mechanics and Analysis, 89, pages 21-86, 1985.

[16] J.-Y. Chemin et C.-J. Xu, Inclusions de Sobolev en calcul de Weyl-Hörmander et systèmes sous-elliptiques. Annales Scientifiques de l'École Normale Supérieure, 30, pages 719-751, 1997.

[17] A. Cohen, Sur la route des ondelettes. Gazette des Mathématiciens, 130, pages 19-36, 2011.

[18] H. Fujita and T. Kato, On the Navier-Stokes initial value problem I. Archive for Rational Mechanics and Analysis, 16, pages 269-315, 1964.

[19] P. Gérard, Description du défaut de compacité de l'injection de Sobolev. ESAIM Contrôle Optimal et Calcul des Variations, $\mathbf{3}$, pages 213-233, 1998.

[20] P. Gérard, Y. Meyer et F. Oru, Inégalités de Sobolev précisées. Séminaire X-EDP, École Polytechnique, 1996.

[21] J. Ginibre and G. Velo, Generalized Strichartz inequalities for the wave equations. Journal of Functional Analysis, 133, pages 50-68, 1995.

[22] L. Hörmander, The Analysis of Linear Partial Differential Equations 3. Springer Verlag, 1985.

[23] S. Jaffard, Analysis of the lack of compactness in the critical Sobolev embeddings. Journal of Functional Analysis, 161, pages 384-396, 1999.

[24] M. Keel and T. Tao, Endpoint Strichartz estimates. American Journal of Mathematics, 120, pages 955-980, 1998.

[25] C. E. Kenig and F. Merle, Global well-posedness, scattering and blow-up for the energy critical focusing non-linear wave equation. Acta Mathematica, 201, pages 147-212, 2008.

[26] S. Klainerman and I. Rodnianski, Rough solutions of the Einstein-vacuum equations. Annals of Mathematics, 161, pages 1143-1193, 2005.

[27] P.-L. Lions, The concentration-compactness principle in the calculus of variations. The limit case. I. Revista Matematica Iberoamericana 1, pages 145-201, 1985.

[28] P.-L. Lions, The concentration-compactness principle in the calculus of variations. The limit case. II. Revista Matematica Iberoamericana 1 , pages 45-121, 1985.

[29] J. Littlewood and R. Paley, Theorems on Fourier series and power series I. Journal of the London Mathematical Society, 6, pages 230-233, 1931.

[30] J. Littlewood and R. Paley, Theorems on Fourier series and power series II. Proceedings of the London Mathematical Society, 42, pages 52-89, 1936.

[31] Stéphane Mallat, A Wavelet Tour of Image Processing: The Sparse Way. Academic Press, 2008

[32] Y. Meyer, Ondelettes et opérateurs. I. Hermann, 1990.

[33] Y. Meyer, Ondelettes et opérateurs. II. Hermann, 1990.

[34] W. Rudin, Fourier Analysis on Groups, Interscience Tracts in Pure and Applied Mathematics, 12, New York-London, 1962. 
[35] B. Ruf, A sharp Trudinger-Moser type inequality for unbounded domains in $\mathbb{R} 2$. Journal of Functional Analysis, 219, pages 340-367, 2005.

[36] L. Schwartz, Théorie des distributions. Hermann, 1966.

[37] R. Strichartz, Restriction Fourier transform of quadratic surfaces and decay of solutions of the wave equations, Duke Mathematical Journal, 44, pages 705-714, 1977.

[38] D. Tataru, Strichartz estimates for operators with non smooth coefficients and the non-linear wave equation. American Journal of Mathematics, 122, pages 349-376, 2000.

[39] D. Tataru, Strichartz estimates for second order hyperbolic operators with non smooth coefficients II. American Journal of Mathematics, 123, pages 385-423, 2000.

[40] H. Triebel, Theory of Function Spaces. Birkhäuser, Basel, 1983.

[41] N. S. Trudinger, On imbedding into Orlicz spaces and some applications. Journal of Mathematics and Mechanics, 17, pages 473-484, 1967.

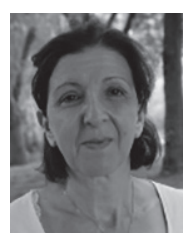

Hajer Bahouri (hajer.bahouri@math.cnrs. fr), Directrix of Research at the National Center for Scientific Research in France, is interested in developing harmonic and microlocal analysis tools to study evolution nonlinear partial differential equations on physical and abstract backgrounds.

This is an English translation by Fernando Pestana da Costa of the French article La théorie de Littlewood-Paley: fil conducteur de nombreux travaux en analyse non linéaire by Hajer Bahouri, published in La Gazette des Mathématiciens, (No. 154, pp. 28-39, Octobre 2017). The EMS Newsletter thanks the author, La Gazette des Mathématiciens, and the Société Mathématique de France for authorisation to republish this article. 